\title{
Corticotropin releasing hormone promotes inflammatory bowel disease via inducing intestinal macrophage autophagy
}

\author{
Sheng-Bing Zhao $\mathbb{D}^{1,3}$, Jia-Yi Wu ${ }^{1,3}$, Zi-Xuan He ${ }^{1,3}$, Yi-Hang Song ${ }^{1,3}$, Xin Chang ${ }^{1,2,3}$, Tian Xia ${ }^{1}$, Xue Fang ${ }^{1}$, Zhao-Shen Li (iD ${ }^{1 凶}$, \\ Can Xu iD ${ }^{1 凶}$, Shu-Ling Wang (D) ${ }^{1 凶}$ and Yu Bai $\mathbb{D}^{1 凶}$
}

(c) The Author(s) 2021

Psychosocial stress is a vital factor contributing to the pathogenesis and progression of inflammatory bowel disease (IBD). The contribution of intestinal macrophage autophagy to the onset and development of IBD has been widely studied. Herein, we investigated the underlying mechanism of psychosocial stress in an IBD mouse model pertaining to macrophage autophagy. Corticotropin releasing hormone $(\mathrm{CRH})$ was peripherally administrated to induce psychosocial stress. For in vivo studies, dextran sulfate sodium (DSS) was used for the creation of our IBD mouse model. For in vitro studies, lipopolysaccharide (LPS) was applied on murine bone marrow-derived macrophages (BMDMs) as a cellular IBD-related challenge. Chloroquine was applied to inhibit autophagy. We found that $\mathrm{CRH}$ aggravated the severity of DSS-induced IBD, increasing overall and local inflammatory reactions and infiltration. The levels of autophagy in intestinal macrophages and murine BMDMs were increased under these IBD-related inflammatory challenges and CRH further enhanced these effects. Subsequent administration of chloroquine markedly attenuated the detrimental effects of $\mathrm{CRH}$ on IBD severity and inflammatory reactions via inhibition of autophagy. These findings illustrate the effects of peripheral administration of CRH on DSS-induced IBD via the enhancement of intestinal macrophage autophagy, thus providing a novel understanding as well as therapeutic target for the treatment of IBD.

Cell Death Discovery (2021)7:377; https://doi.org/10.1038/s41420-021-00767-8

\section{INTRODUCTION}

Inflammatory bowel disease (IBD), which includes ulcerative colitis (UC) and Crohn's disease (CD), is a group of diseases characterized by the chronic and recurrent inflammation of intestinal mucosa $[1,2]$. In UC, the areas of inflammation extend continuously from the rectum throughout the whole colon, and inflammation is mainly the mucosa. In contrast, inflammation in CD is characterized by patchy "skip lesions" in the distal small intestine and colon, which are transmural and accompanied by lymphoid aggregation $[3,4]$. IBD pathogenesis is widely acknowledged to involve an abnormality in the intestinal defense system, comprising the mucosa, intestinal epithelial cells, and subendothelial immune cells, ultimately resulting in damage to the intestinal mucosal barrier and a disturbance in microbial homeostasis $[5,6]$. Considering the potential factors responsible for the induction of intestinal defense system damage, the accumulation and activation of macrophages in the submucosa layer contributes greatly to the overinduction of various forms of inflammatory and immune responses $[7,8]$. Thus, regulating macrophage-induced intestinal inflammatory and immune responses may be an effective approach in the treatment of IBD.

Psychosocial stress has been reported to be a contributing factor in the pathogenesis and progression of IBD, eliciting damage to the intestinal defense system and disturbing microbial homeostasis [9-11]. In central nervous system, the physiological response to stress lies in the triggering of hypothalamic-pituitary-adrenal axis, thus releasing corticotropin releasing hormone $(\mathrm{CRH})$ for the regulation of neuroendocrine function and immune reaction $[12,13]$. CRH is predominantly synthesized in the paraventricular nucleus of the hypothalamus and secreted into portal capillaries converging at the anterior lobe of the pituitary gland [14]. In addition, CRH is also widely expressed in extracranial tissues, including the endometrium, placenta, liver, stomach, and small and large intestines, but at much lower levels than that in the hypothalamus $[15,16]$. Nonetheless, recent studies have revealed that peripheral $\mathrm{CRH}$ can also play a key role in stress-induced intestinal disturbances. In the intestine, $\mathrm{CRH}$ and $\mathrm{CRH}$-related peptides, such as urocortin 1, 2, and 3, are produced by enterochromaffin cells, Paneth cells, colonocytes, mast cells, and mucosal macrophages. These peptides are also secreted by the myenteric plexus and submucosal plexus [17-19]. CRH in peripheral tissues can directly regulate the intestinal inflammatory and immune responses through $\mathrm{CRH}$ receptor 1 (CRHR1) and CRHR2 in the digestive tract, via so-called "brain-gut interactions" [20, 21]. Although peripheral $\mathrm{CRH}$ has been demonstrated to play an important role in the onset and development of IBD, the underlying mechanisms have not been elucidated $[22,23]$.

Autophagy is a vital metabolic mechanism involving the degradation of misfolded proteins and damaged cellular organelles in lysosomes for subsequent recycling [24, 25]. The formation of autophagosomes and their fusion with lysosomes

\footnotetext{
${ }^{1}$ Department of Gastroenterology, Changhai Hospital, Second Military Medical University/Naval Medical University, Shanghai, China. ${ }^{2}$ Department of Gastroenterology, General Hospital of Central Theater Command, Wuhan, China. ${ }^{3}$ These authors contributed equally: Sheng-Bing Zhao, Jia-Yi Wu, Zi-Xuan He, Yi-Hang Song, Xin Chang. ${ }^{凶}$ email: li.zhaoshen@ @hotmail.com; xxcc211@126.com; wangshuling0000@126.com; baiyu1998@hotmail.com
}

Received: 26 May 2021 Revised: 7 November 2021 Accepted: 15 November 2021

Published online: 07 December 2021 
to form autolysosomes is regarded as the onset of the autophagy process, and this process is termed "autophagy flux" [26]. Autophagy has been reported to be involved in the pathogenesis and progression of various diseases. In the digestive system, it was previously demonstrated that baseline autophagy is crucial for the maintenance of intestinal homeostasis, and autophagy dysfunction may play a role in the onset and aggravation of IBD [27, 28]. Conversely, abnormal or overwhelming induction of intestinal macrophage autophagy has been reported to contribute to the aggravation of IBD, ostensibly through the triggering of inflammatory responses and reactive oxidative stress [29,30]. Hence, additional studies are necessary to explore the complex role of autophagy in IBD.

In the present study, we peripherally applied $\mathrm{CRH}$ to mimic psychosocial stress in a dextran sulfate sodium (DSS)-induced IBD mouse model [31]. We hypothesize that psychosocial stress contributes to the aggravation of IBD through the enhancement of intestinal macrophage autophagy. By advancing our understanding of the role of autophagy in IBD, an effective therapeutic strategy for the treatment of IBD may be revealed.

\section{RESULTS}

\section{CRH aggravated the severity of IBD}

To determine the influence of psychosocial stress on IBD, peripheral administration of $\mathrm{CRH}$ was used to reproduce the effect of psychosocial stress in a mouse model of IBD. To generate the mouse model, C57BL/6 mice were consecutively treated with $3 \%$ DSS for 6 days. The effects of this treatment included sustained weight loss (Fig. 1A), increased DAl score (Fig. 1B), and shortened colon length (Fig. 1C, D). Subsequent administration of $\mathrm{CRH}$ to DSS-treated mice further aggravated these IBD symptoms (Fig. $1 A-D)$. While levels of proinflammatory cytokines, tumor necrosis factor- $a$ (TNF-a) and interleukin (IL)-18, were elevated in the serum of IBD mice, these levels were further elevated by CRH treatment (Fig. 2A). A histological morphology analysis of inflammatory infiltration in the left colon also revealed increased inflammation (Fig. 2B, C). Furthermore, while mRNA expression levels of proinflammatory factors in the colon, including TNF-a, IL-18, and IL-1 $\beta$, were significantly elevated in IBD mice, the mRNA expression levels were further increased by CRH (Fig. 2D). Together, these results provide evidence that peripheral administration of a psychosocial stress mimic agent (CRH) could exacerbate the development of IBD.

Antalarmin and astressin2-B were applied to selectively block the CRHR1 and CRHR2 and the effect of CRH on the DSS model mice determined (Supplementary Fig. 1). While CRH administration aggravated the severity of IBD-associated body weight loss, DAI score, change in colon length, and inflammatory infiltration in the left colon, these effects were blocked by the application of antalarmin (Supplementary Fig. 1A-C, G, H). In contrast, astressin2$\mathrm{B}$ administration did not significantly affect $\mathrm{CRH}$-induced IBDassociated body weight loss, DAl score, change in colon length, and inflammation infiltration (Supplementary Fig. 1D-H).

In our previous study, we demonstrated that psychosocial stress was positively associated with levels of autophagy markers in intestinal samples [32]. To verify that intestinal macrophage autophagy mediated the inflammation induced by $C R H$ in IBD, we repeated our analysis in another 12 IBD patients, and observed a similar trend (Supplementary Fig. 2). We recruited six patients with $\mathrm{mild} /$ moderate IBD and six patients with severe IBD. The baseline characteristics, including sex, age, disease duration, smoking history, and medications, were well balanced between the two groups; severe IBD patients showed higher CPSS scores and Mayo scores, both of which were positively related (Supplementary Fig. $2 \mathrm{~A}, \mathrm{~B})$. The endoscopic images, histological results (H\&E staining), monocyte/macrophage infiltration (CD68 immunohistochemistry) results, and autophagy results (light chain 3, LC3 immunohistochemistry) for these patients were shown in Supplementary Fig. 2C. All above indicators were significantly increased in the colons of patients with higher reported CPSS (compared with patients with lower levels of perceived stress). In addition, the LC3-II/I ratios in intestinal biopsy specimens from patients with $\mathrm{mild} /$ moderate IBD were higher than those in healthy control individuals (whose basic characteristics were matched). Moreover, the LC3-II/I ratios in intestinal biopsy specimens from severe IBD patients were markedly higher. In contrast, p62/SQSTM1 marker levels were reduced, which indicated that autophagy levels were higher in IBD patients (Supplementary Fig. 2D). As shown in Supplementary Fig. 2E, autophagy levels were also positively correlated with CPSS and the modified Mayo scores.

\section{Peripheral administration of CRH further increased intestinal autophagy levels in IBD}

High autophagy levels have been reported to play a role in the pathogenesis and progression of IBD [28, 32, 33]. In the present study, we investigated whether administration of $\mathrm{CRH}$ affected levels of autophagy in colonic tissues. After 6 days of treatment with $3 \%$ DSS, we observed an increase in Beclin-1 levels, an increase in the LC3-II/I ratio, and a decrease in p62/SQSTM1 levels in IBD model mice (Fig. 3A, B). These three markers of colonic autophagy were further changed after subsequent $\mathrm{CRH}$ administration (Fig. 3A, B). To further investigate these results, we also used immunofluorescence to evaluate the expression of autophagy markers, and the results demonstrated a similar increasing trend in the number of LC3 dots in the left colon (Fig. 3C). In addition, we labeled mouse macrophages via the F4/80 macrophage-specific marker and found that the numbers of F4/ 80 dots were significantly increased in the DSS + CRH group (compared to the DSS group). Moreover, the F4/80 dots were observed to colocalize with LC3 dots. Taken together, these results provide evidence that peripheral $\mathrm{CRH}$ further enhances intestinal autophagy in IBD, specifically colonic macrophage-associated autophagy.

\section{CRH administration further enhanced autophagy levels in bone marrow-derived macrophages (BMDMs) under the challenge of lipopolysaccharide (LPS)}

Since macrophages also play an important role in the pathogenesis and progression of IBD through activation of the innate immune system, activation of the acquired immune system, and by triggering the production of proinflammatory factors, we further explored the effects of $\mathrm{CRH}$ on autophagy in BMDMs under the challenge of LPS in vitro. The mRNA expression levels of IL-1 $\beta$, IL-18, and TNF-a were significantly elevated in BMDMs treated with LPS, and these levels were further increased by CRH (Fig. 4A). In addition, levels of Beclin-1 and the LC3-II/I ratio were increased, and the level of p62 was decreased, in BMDMs stimulated by LPS (compared with the normal group). Moreover, administration of $\mathrm{CRH}$ markedly enhanced the levels of these autophagy-related indicators (Fig. 4B, C). Similar trends were observed in the numbers of autophagosomes in BMDMs (Fig. 4D, E) and in the autophagy flux (Fig. 4F, G). These results demonstrate that $\mathrm{CRH}$ further enhances the level of autophagy in BMDMs under the challenge of LPS.

When BMDMs were cocultured with colonic organoids in vitro, the activated macrophages negatively affected intestinal-derived cells (Supplementary Fig. 3). First, BMDMs were plated on the top membrane of transwell inserts and incubated for a minimum of $12 \mathrm{~h}$ with or without LPS. When a cell attachment rate of $60 \%$ was achieved, the plated BMDMs were cocultured with mouse colonic organoids. In comparison to cocultures with resting macrophages, the growth rate of mouse colonic organoids, shown by morphological changes and the number of PCNA-positive fluorescence points, was significantly decreased in cocultures with LPS-activated BMDMs (Supplementary Fig. 3A, B). Moreover, 
A
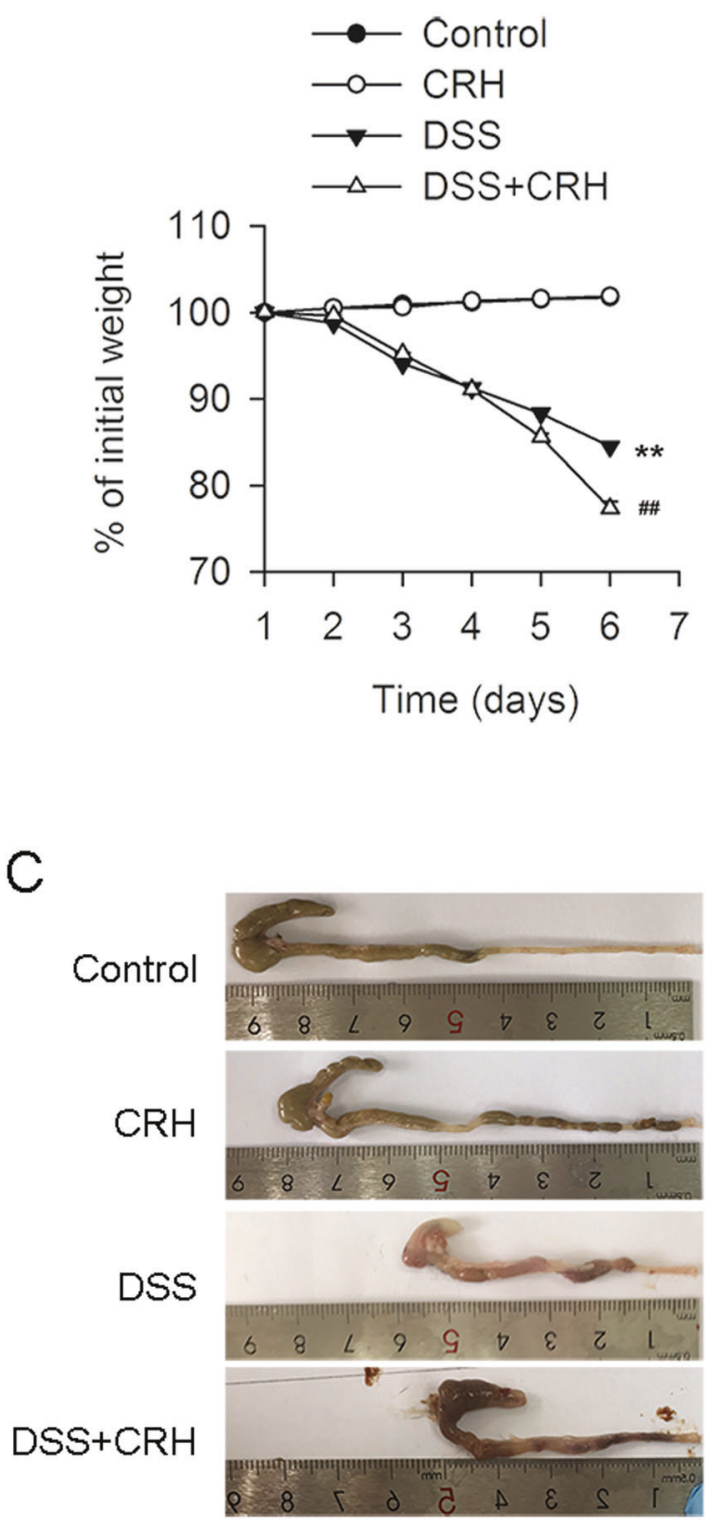

$\mathrm{B}$
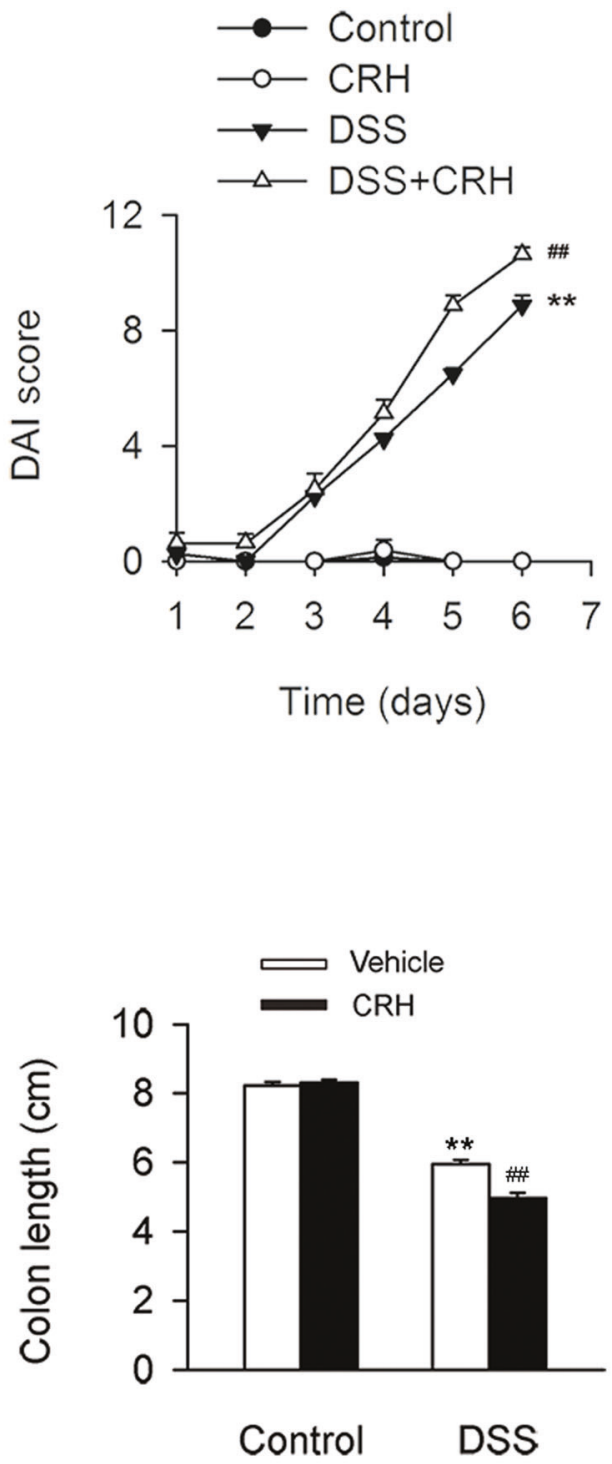

Fig. 1 Peripheral administration of CRH contributed to the deterioration of IBD. C57BL/6 mice were administered DSS (3\%) for 6 days (and a control group was provided with water only for comparison). For the $\mathrm{CRH}$ treatment group, $\mathrm{CRH}$ (or vehicle only) was intraperitoneally injected at a dose of $50 \mu \mathrm{g} / \mathrm{kg}$ body weight from day 1 to day 6 using saline as the vehicle. Body weight loss, stool consistency, occult or gross blood per rectum, and colon length were then recorded by two researchers who were blinded to the treatments. A-D In comparison with the DSS + vehicle group, mice in the DSS + CRH group demonstrated a significant aggravation of IBD-associated changes in body weight, DAI score, and colon length ( $n=8$ per group). ${ }^{* *} P<0.01$ vs. the control group; ${ }^{\# \#} P<0.01$ vs. the DSS + vehicle group.

the intestinal mucosal barrier, shown by the number of $\mathrm{E}$ cadherin-positive fluorescence points, was significantly decreased in the colonic organoids cocultured with LPS-activated macrophages (Supplementary Fig. 3B).

\section{Induction of autophagy by rapamycin aggravated CRH- induced colonic damage in an IBD mouse model}

The effects of autophagy on CRH-induced intestinal damage were also examined following rapamycin induction of autophagy (Supplementary Fig. 4). IBD-associated body weight loss, DAI score, change in colon length, and inflammatory infiltration in the left colon were all significantly aggravated in the DSS + rapamycin group (in comparison with the DSS group). Furthermore, mice in the DSS $+\mathrm{CRH}$ group showed aggravated IBD symptoms (compared with the DSS + vehicle group), but these symptoms were not significantly modulated by rapamycin.

\section{Blockade of autophagy attenuated CRH-induced colonic damage in an IBD mouse model}

To investigate the role of autophagy in $\mathrm{CRH}$-induced intestinal damage in IBD mice, we used chloroquine, which blocks the binding of autophagosomes to lysosomes, to elicit a pharmacological blockade of the autophagy process and subsequently evaluated the severity of IBD. While peripheral administration of $\mathrm{CRH}$ aggravated IBD-associated body weight loss, DAI score, and change in colon length, blocking the autophagy process with chloroquine significantly alleviated IBD-associated changes in body weight loss and DAl score, but not the change in colon 
A

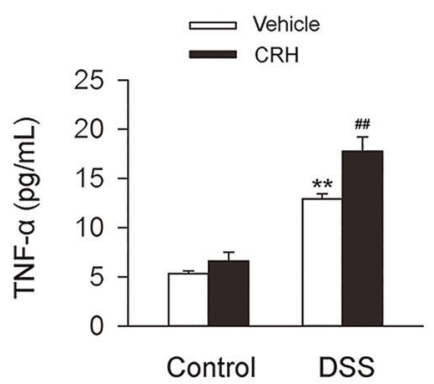

C

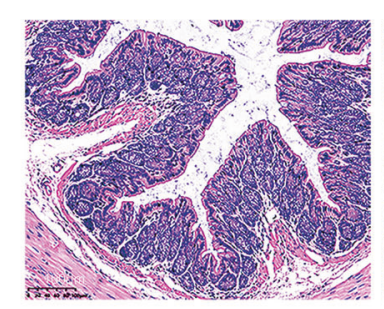

Control

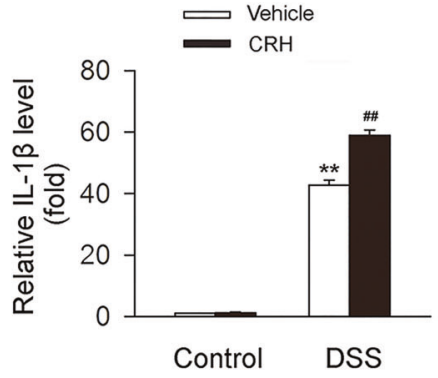

$\mathrm{CRH}$

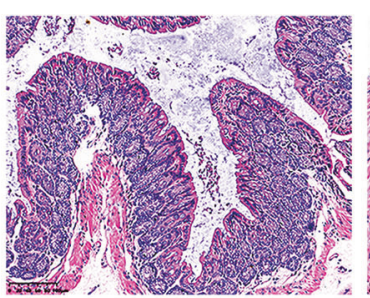

B

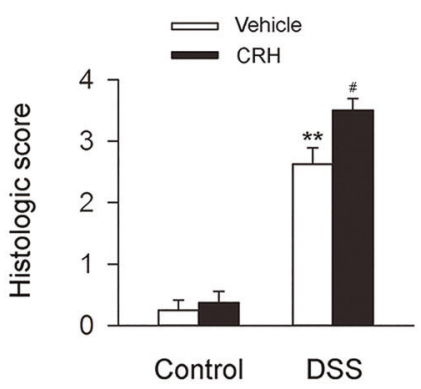

D

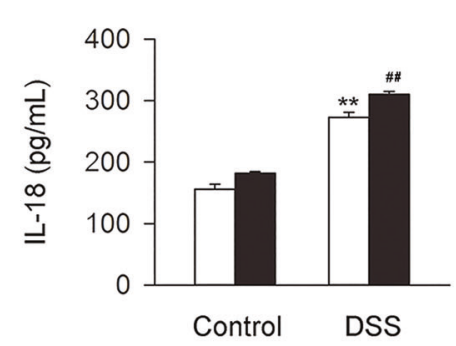

DSS
DSS+CRH

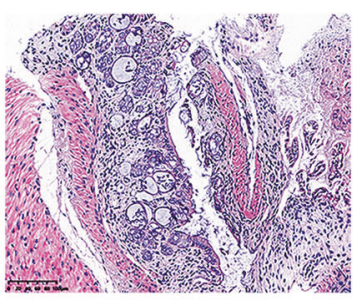

$\sin$
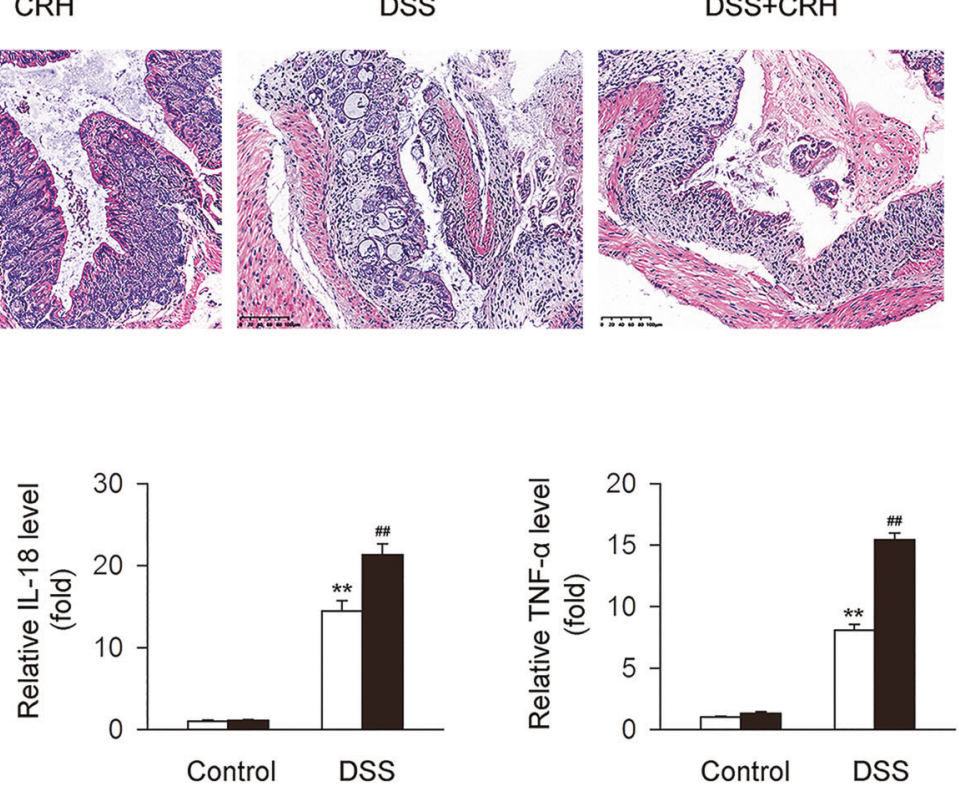

Fig. 2 CRH contributed to the increase of inflammation in IBD mice. C57BL/6 mice were administered DSS (3\%) for 6 days (and a control group was provided with water only for comparison). For the CRH treatment group, $\mathrm{CRH}$ (or vehicle only) was intraperitoneally injected at a dose of $50 \mu \mathrm{g} / \mathrm{kg}$ body weight from day 1 to day 6 using saline as the vehicle. A In comparison with the DSS + vehicle group, mice in the DSS + CRH group demonstrated increased levels of TNF- $\alpha$ and IL-18 in serum ( $n=6$ per group). ${ }^{* *} P<0.01$ vs. the control group; ${ }^{*} P<0.05$ vs. the DSS + vehicle group; ${ }^{\# \#} P<0.01$ vs. the DSS + vehicle group. B, C The left edge of the left colon was separated and fixed, and then H\&E staining was used for the detection of inflammatory infiltration (which was assessed using a histological score). In comparison with DSSinduced colitis mice, mice in the DSS + CRH group demonstrated a significant aggravation of inflammation in the left colon ( $n=8$ per group). ${ }^{* *} P<0.01$ vs. the control group; ${ }^{\#} P<0.05$ vs. the DSS + vehicle group. D IL-1 $\beta$, IL-18, and TNF- $\alpha$ mRNA levels in left colon tissues were analyzed by real-time qPCR. CRH administration also further enhanced levels of these proinflammatory factors in DSS-induced mice ( $n=6$ per group). ${ }^{*} P<0.01$ vs. the control group; ${ }^{\# \#} P<0.01$ vs. the DSS + vehicle group.

length (Fig. 5A-C). In addition, chloroquine suppressed inflammatory infiltration in the left colon resulting from DSS-induced colitis (Fig. 5D, E). Chloroquine also attenuated CRH-induced enhancement of autophagy in the left colon, reduced the number of macrophages, and reduced the number of LC3 dots that colocalized with F4/80 dots (Fig. 6). These data provide evidence that autophagy is required for $\mathrm{CRH}$-induced colonic damage in our IBD mouse models.

The role of autophagy in $\mathrm{CRH}$-induced intestinal damage in IBD mice was also verified using 3-MA, another autophagy inhibitor. While CRH significantly aggravated the severity of IBD (as assessed by IBD-associated body weight loss, DAI score, change in colon length, and inflammatory infiltration), 3-MA administration significantly attenuated the detrimental effects of peripheral administration of $\mathrm{CRH}$ on body weight loss and DAl score, but not change in colon length (Supplementary Fig. 5A-C). Moreover, 3-MA significantly alleviated inflammation in the DSS $+\mathrm{CRH}+3-$ MA group (in comparison with the DSS + CRH group) (Supplementary Fig. 5D, E). Together, these results indicate the potential therapeutic effects of autophagy blockade in peripheral $\mathrm{CRH}$ induced IBD.

\section{Chloroquine attenuated CRH-induced increases in inflammation and autophagy in BMDMs under the challenge of LPS}

Finally, we investigated whether $\mathrm{CRH}$ could induce an increase in inflammation via the process of autophagy in BMDMs under inflammatory loading in vitro. In murine BMDMs under the challenge of LPS, CRH administration further increased the mRNA and protein expression levels of proinflammatory cytokines, including IL-1 $\beta$, TNF- $\alpha$, and IL-18. However, these effects of CRH were attenuated following blockade of the autophagy process by chloroquine (Fig. 7A, B). In addition, chloroquine attenuated CRHinduced increases in autophagy-related protein levels and autophagy flux in LPS-treated BMDMs (Fig. 8). Taken together, these data provide evidence that autophagy plays a role in the $\mathrm{CRH}$-induced enhancement of inflammation in BMDMs under the challenge of LPS. 
A

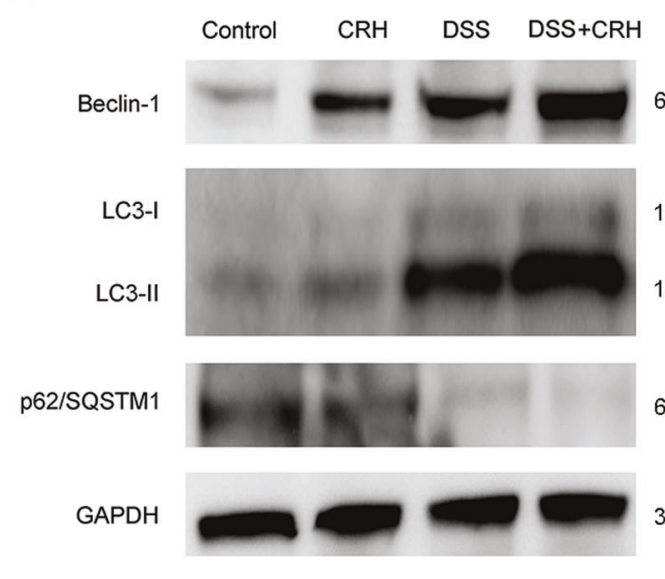

B

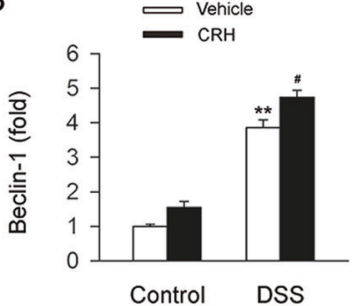

$16 \mathrm{KD}$ 6OKD

$37 \mathrm{KD}$
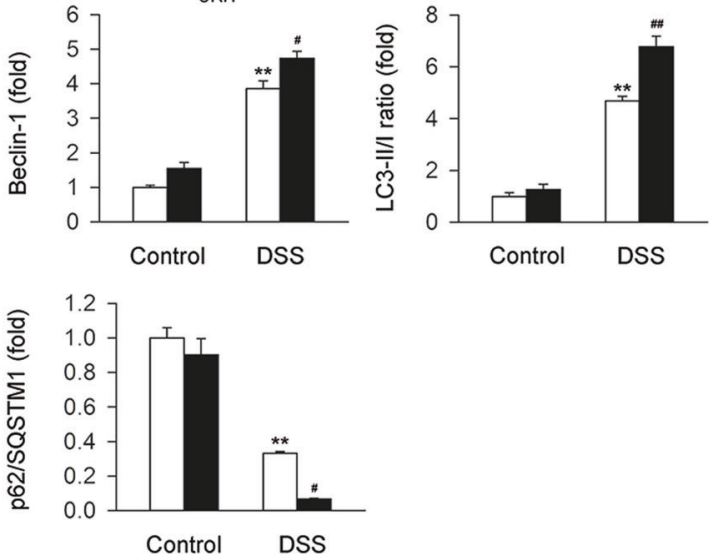

C
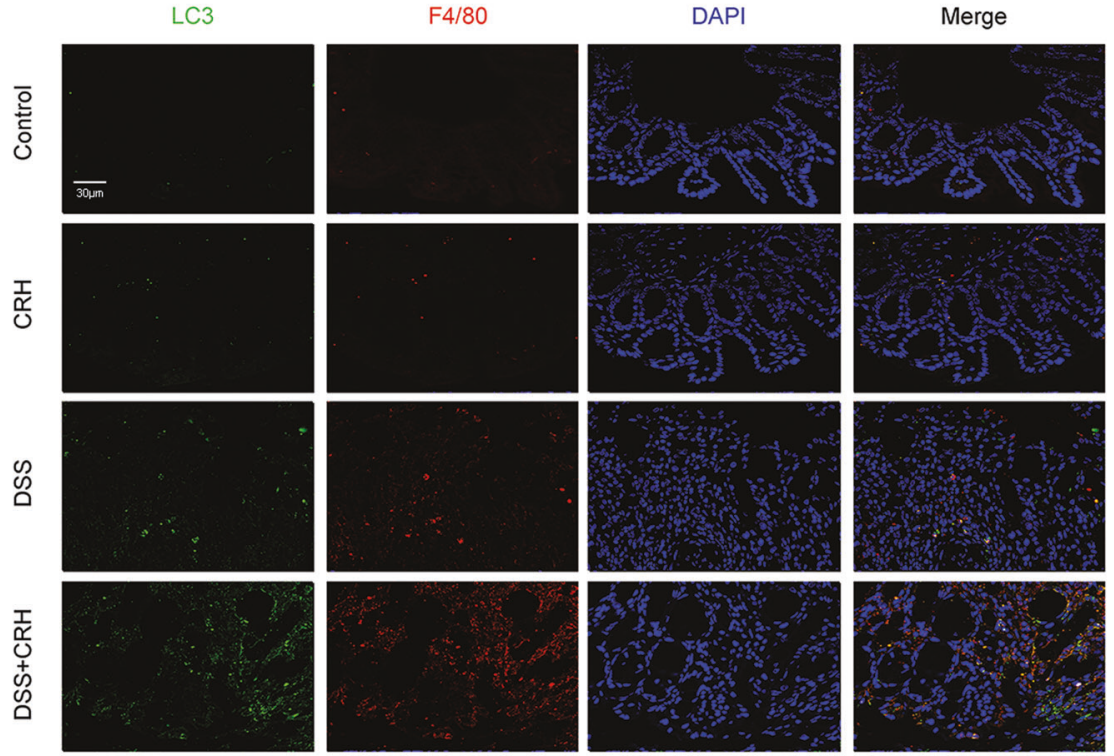

Fig. 3 Intestinal autophagy in IBD was further enhanced by CRH. C57BL/6 mice were administered DSS (3\%) for 6 days (and a control group was provided with water only for comparison). For the CRH treatment group, CRH (or vehicle only) was intraperitoneally injected at a dose of $50 \mu \mathrm{g} / \mathrm{kg}$ body weight from day 1 to day 6 using saline as the vehicle. A, B The levels of autophagy-related proteins, including Beclin-1, the LC3-II/I ratio, and p62/SQSTM1, were detected by western blot. In comparison with the DSS + vehicle group, mice in the DSS + CRH group exhibited significantly increased levels of Beclin-1 and the LC3-II/I ratio, but a decrease of p62 $\left(n=5\right.$ per group). ${ }^{* *} P<0.01$ vs. the control group; ${ }^{\#} P<0.05$ vs. the DSS + vehicle group; ${ }^{\# \# ~} P<0.01$ vs. the DSS + vehicle group. $C$ The left edge of the left colon was separated and fixed and then H\&E staining was used for the detection of inflammatory infiltration (which was assessed using a histological score). An increase was observed in the number of LC3 dots and the number of macrophages (which were colocalized).

\section{DISCUSSION}

Here, we report for the first time that intestinal macrophage autophagy is highly associated with psychosocial stress and that it promotes the pathogenesis and progression of IBD. First, we demonstrated that peripheral administration of CRH to mimic psychosocial stress induced a detrimental effect on IBD severity, and enhanced both overall and local inflammatory reactions and infiltration in a DSS-induced IBD mouse model. Autophagy, a vital metabolic mechanism, was found to be induced following the administration of $\mathrm{CRH}$ in macrophages both in vivo (colonic issues) and in vitro (murine BMDMs under IBD-related inflammatory stimulation). Furthermore, chloroquine application for the inhibition of overinduced autophagy alleviated IBD-associated effects in DSS-induced IBD aggravated by CRH.

Psychosocial stress is known to enhance the pathogenesis and progression of IBD by suppressing the intestinal defense system [9-11]. In general, the physiological stress response involves the triggering of the hypothalamic-pituitary-adrenal axis, thus releasing $\mathrm{CRH}$ for the regulation of neuroendocrine function and internal organ immune activities. However, $\mathrm{CRH}$ is also secreted by peripheral tissues in the digestive system and by immune cells, including Paneth cells, macrophages, and mast cells [17-19]. Elevated CRH levels damage the mucosal barrier and enhance colonic hyperpermeability, ultimately resulting in stress-related intestinal disorders [20]. Recent studies reveal that peripheral CRH plays an important role in IBD [34-37]. Indeed, the mucosal epithelial cells of colonic tissues from IBD patients have been shown to express high amounts of CRH (both protein and mRNA).

The effects of colonic CRH on IBD pathogenesis may potentially be modulated [34]. Systemic CRH deficiency reduced local inflammatory responses in colonic tissues in an experimental colitis mouse model [36]. Furthermore, peripheral CRH enhanced visceral nociception in recovery from experimental colitis models [35]. Chen et al. [37] previously reported that consecutive 
A
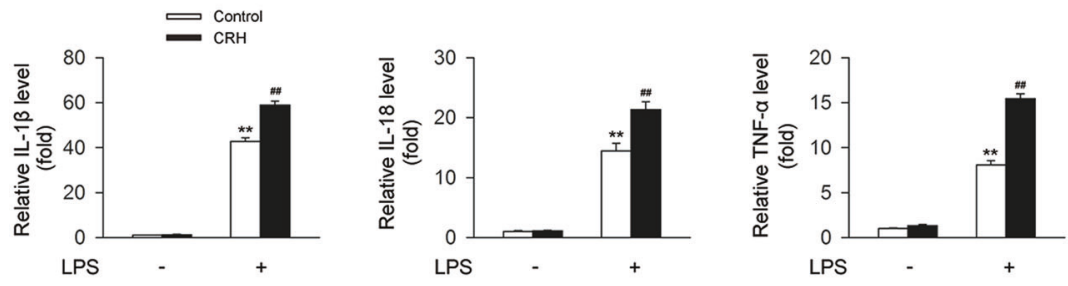

B

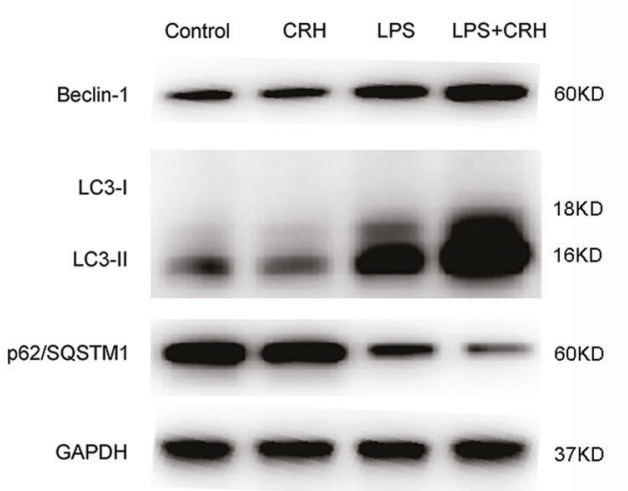

D
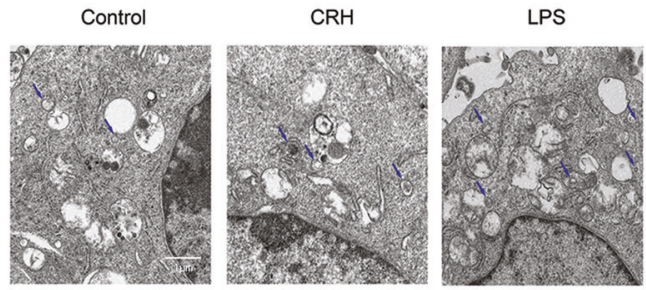

$\mathrm{F}$
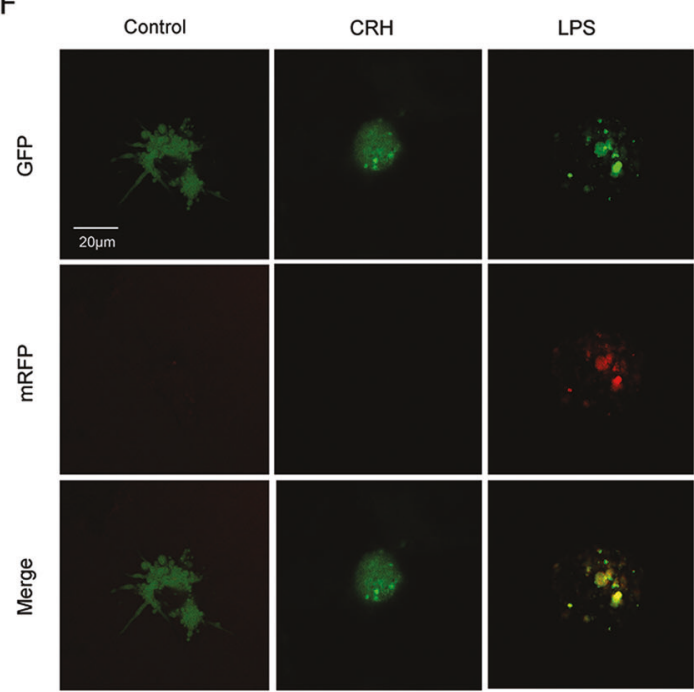
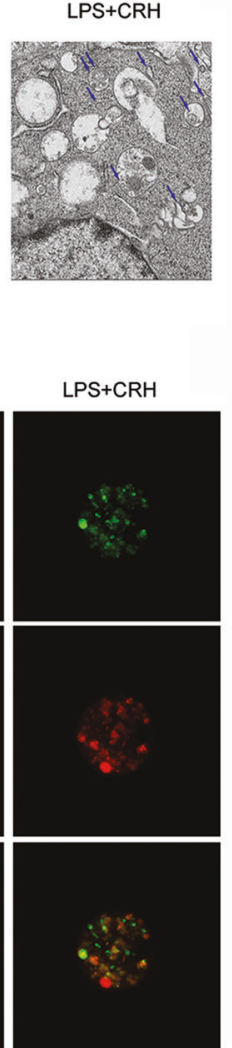

C
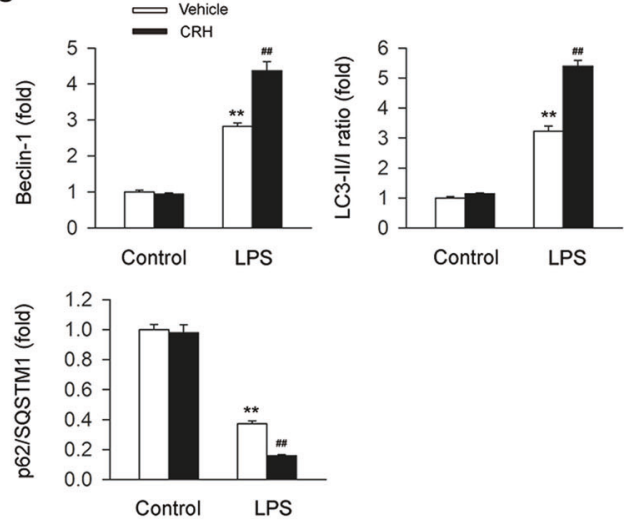

$\mathrm{E}$

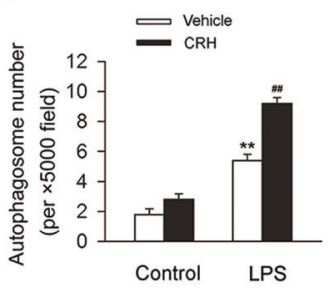

G
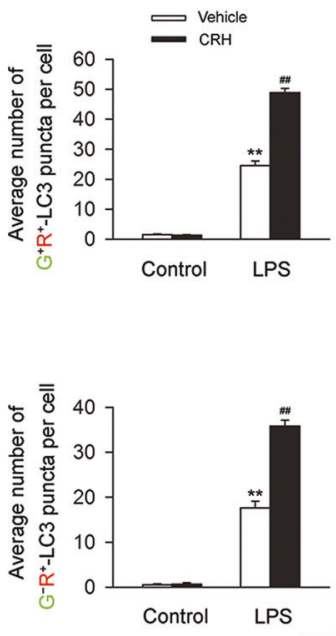

administration of $\mathrm{CRH}$ in a DSS-induced mouse model aggravated IBD. Moreover, Teitelbaum et al. revealed that peripheral administration of $\mathrm{CRH}$-induced chronic stress effects and intestinal barrier dysfunction [38]. Similarly, Vanuytsel et al. reported that peripheral $\mathrm{CRH}$ administration reproduced the effects of psychosocial stress and triggered the onset and development of IBD [31]. CRHR1 antagonists have been proven to be effective in the alleviation of IBD via the blockade of colonic hypersensitivity induced by colonic inflammatory reactions, thus providing a novel therapeutic option for the treatment of stress-aggravated IBD 
Fig. 4 Autophagy in BMDMs challenged with LPS was aggravated by CRH. BMDMs from C57BL/6 mice were isolated and treated with/ without LPS $(100 \mathrm{ng} / \mathrm{mL}) / C R H\left(10^{-8} \mathrm{M}\right)$ for $24 \mathrm{~h}$. IL-1 $\beta$, IL-18, and TNF- $\alpha$ mRNA levels were analyzed by real-time qPCR. The levels of autophagyrelated proteins were analyzed in western blots. Autophagosome number was evaluated using transmission electron microscopy. Autophagy flux was assessed using the mRFP-GFP-LC3 plasmid. A In comparison with LPS-treated BMDMs, BMDMs treated with LPS + CRH exhibited a significant increase in the mRNA levels of proinflammatory cytokines ( $n=6$ per group). ${ }^{* *} P<0.01$ vs. the control group; ${ }^{\# \#} P<0.01$ vs. the LPS + vehicle group. B, C In comparison with the LPS + vehicle group, BMDMs in the LPS + CRH group demonstrated an increase in Beclin-1, an increase in the LC3-II/I ratio, and a decrease in p62/SQSTM1 ( $n=5$ per group). ${ }^{* *} P<0.01$ vs. the control group; ${ }^{\# \#} P<0.01$ vs. the LPS + vehicle group. An increase in the number of autophagosomes $(\mathbf{D}, \mathbf{E})(n=5$ per group) and an increase in autophagy flux $(\mathbf{F}, \mathbf{G})$ were also observed ( $n=6$ per group). ${ }^{* *} P<0.01$ vs. the control group; ${ }^{\# \#} P<0.01$ vs. the LPS + vehicle group.

$[22,36,37,39]$. In agreement with these results, we found that $\mathrm{CRH}$ administration markedly aggravated the severity of IBD, enhancing body weight loss, DAl score, colon length shortening, and inflammatory infiltration. Moreover, these effects were markedly blocked by the selective CRHR1 antagonist antalarmin. In contrast, astressin2- $B$, a selective CRHR2 antagonist, had no significant effect on IBD. Together, these data provide evidence that macrophage-induced inflammatory reactions play an important role in $\mathrm{CRH}$-mediated aggravation of IBD.

Autophagy is an important cellular physiological pathway for the degradation of long-lived proteins and damaged organelles into metabolic products and their subsequent recycling to maintain cellular homeostasis [40, 41]. The autophagy process involves the formation of autophagosomes containing sequestered cytoplasmic materials and their subsequent fusion with lysosomes to form functional autolysosomes. Although baseline autophagy was considered to be important in the maintenance of intestinal homeostasis [28], overinduction of autophagy may aggravate IBD through the induction of autophagic cell death, thus leading to a disruption in the intestinal barrier and the excessive production of proinflammatory cytokines [30, 42]. Chloroquine, a classic autophagy inhibitor, could potentially be repurposed as a therapy for the treatment of IBD, regulating cellular metabolism and modulating inflammatory activities [42-45].

In our in vivo studies, DSS was applied to mimic IBD in a mouse model. Since macrophages are one of the major cells responding to $\mathrm{CRH}$-induced stress in IBD, our specific aim was to study autophagy in macrophages. To complement our in vivo studies, we conducted a series of in vitro studies using isolated macrophages. These in vitro studies provide scientific rigor and a validation for our hypotheses. BMDMs were chosen for these studies because they are widely acknowledged to be a primary macrophage and are known to be suitable for use in vitro studies. Previous studies from our lab (and others) have demonstrated that LPS can be used to mimic DSS-mediated stress in macrophages in in vitro studies $[32,46,47]$. Thus, in vitro studies involving LPS stimulation of BMDMs should provide a dependable model from which we can draw solid conclusions.

Using our in vivo and in vitro models, we demonstrate that macrophage autophagy was significantly increased in colonic tissues in vivo (after DSS-induced IBD) and in murine BMDMs in vitro (after LPS stimulation). In both cases, peripheral administration of CRH further enhanced the levels of macrophage autophagy. In addition, chloroquine inhibition of autophagy was observed in both our DSS-induced IBD mouse model and in murine BMDMs. Chloroquine and 3-MA treatments largely alleviated the detrimental effects of $\mathrm{CRH}$ in DSS-induced mice. However, rapamycin did not significantly aggravate the effect of $\mathrm{CRH}$ on DSS-induced mice. This may be explained by the presence of severe IBD symptoms in the DSS + CRH group and/or the small sample size. Taken together, these findings provide evidence of the involvement of macrophage autophagy in the detrimental effects of $\mathrm{CRH}$ on IBD. Moreover, they demonstrate the therapeutic effect of the blockade of macrophage autophagy in DSS-induced colitis involving peripheral $\mathrm{CRH}$.
In the present study, we reveal that $\mathrm{CRH}$ can promote IBD by inducing intestinal macrophage autophagy. To explore specific autophagy mechanisms in IBD-related macrophages, we previously applied an autophagy-specific microarray to monitor the expression profiles of autophagy genes in our IBD mouse model [32]. We found that the levels of several vital autophagy-related genes and proteins were significantly up-regulated in the DSS $+\mathrm{CRH}$ group when compared to the DSS group. In addition, $\mathrm{CRH}$ increased the levels of Beclin-1, Atg16L1, PIK3R4, and Atg4B proteins, but decreased the levels of GAA, CTSD, and PPKAA1 proteins (in western blot analyses). In consideration of limitations in the contents and theme of our present study, we did not explore further the specific mechanisms underlying macrophage autophagy. We acknowledge that this omission may limit the longitudinal depth and clinical translation of our current work. However, we plan to further investigate these mechanisms in subsequent research.

Taken together, we have demonstrated the aggravation effect of psychosocial stress mediated by peripheral administration of $\mathrm{CRH}$ on DSS-induced IBD via induction of macrophage autophagy. We believe that these findings could further improve our understanding of the pathogenesis and progression of IBD, and provide a novel insight into IBD treatments. However, to ultimately develop effective therapeutic strategies for IBD that target macrophage autophagy, additional mechanistic studies are required.

\section{MATERIALS AND METHODS}

\section{Animal care and use}

C57BL/6 mice were provided by Shanghai Super-B\&K Laboratory Animal Corp., Ltd. (Shanghai, China), and kept at $22^{\circ} \mathrm{C}$ under a 12-h light/dark cycle with free access to water and a standard rodent diet. At the end of the experiments, the mice were anaesthetized with phenobarbital sodium $(60 \mathrm{mg} / \mathrm{kg}$, i.p.) and euthanized using cervical dislocation unless indicated otherwise. All of the procedures were approved and conducted in accordance with the guidelines of the Animal Care Committee of Navy Medical University, Shanghai, China.

\section{Induction of IBD mouse model}

The IBD mouse model was induced in C57BL/6 mice (8-10-week-old males) with 3\% DSS (mol. wt. 36,000-50,000 kDa, MP Biomedicals LLC, Santa Ana, CA, USA) dissolved in drinking water provided ad libitum for 6 days (day 1-day 6). For certain mice, $\mathrm{CRH}(50 \mu \mathrm{g} / \mathrm{kg}$ body weight, Tocris, Ellisville, MO, USA) and/or chloroquine $(60 \mathrm{mg} / \mathrm{kg}$ body weight, Sigma-Aldrich, St. Louis, MO, USA) were intraperitoneally injected from day 1 through day 6 (with saline as a vehicle).

\section{Disease activity score and histological analysis in mice}

Body weight, the presence of occult or gross blood per rectum, stool consistency, and colon length were documented by two researchers blinded to the treatment groups. A scoring system was used to assess diarrhea and the presence of occult or overt blood in the stool [48]. Changes in body weight are reported as percentage loss of baseline body weight. The ring of the rectum was harvested postmortem, fixed in $4 \%$ buffered formalin, and embedded in paraffin. For histological analysis, sections were stained with H\&E according to standard protocols. Histological scoring was evaluated in a blinded protocol by a professional pathologist. Cell infiltration was scored as: 1 , an increase in inflammatory 
A

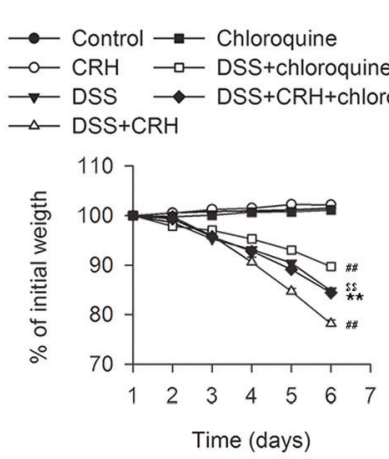

B
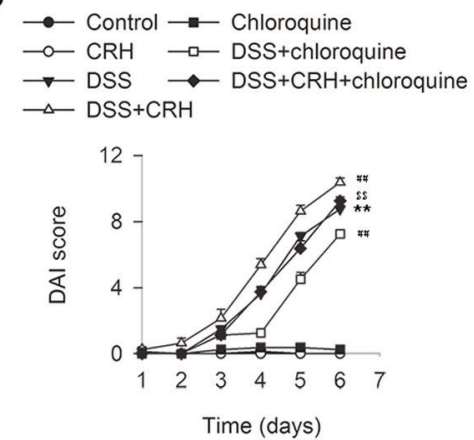

C
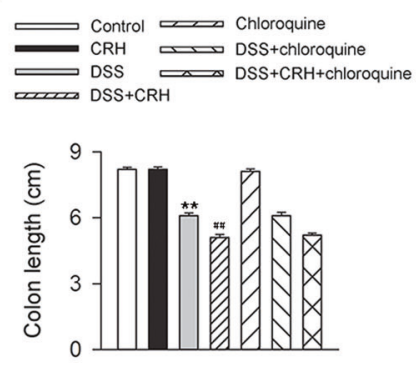

D

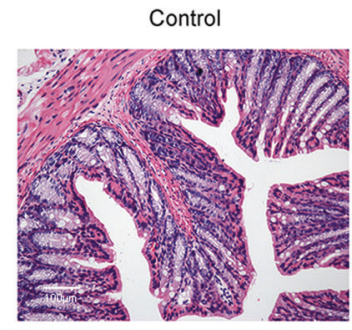

Chloroquine

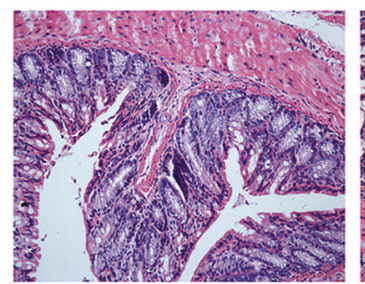

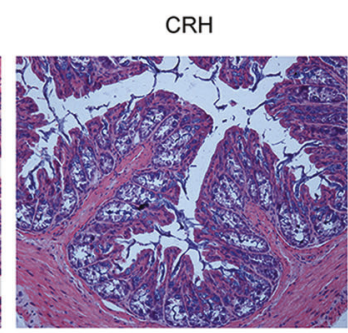

DSS+chloroquine

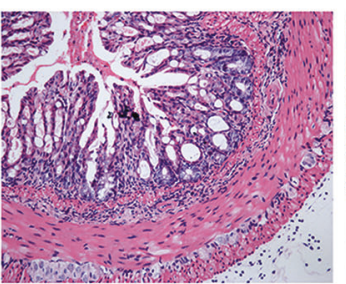

DSS

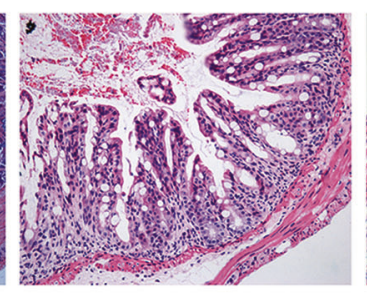

E

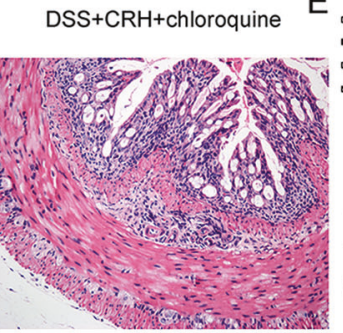

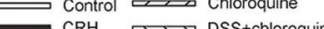
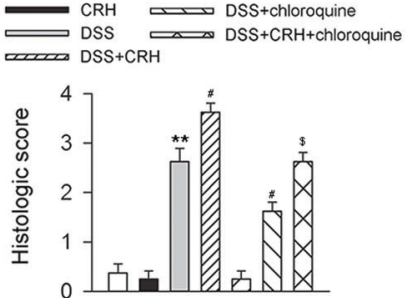

Fig. 5 Chloroquine attenuated CRH-induced colonic damage in IBD mice. C57BL/6 mice were administered DSS (3\%) for 6 days (and a control group was provided with water only for comparison). For the $\mathrm{CRH}$, chloroquine, and $\mathrm{CRH}$-chloroquine groups, $\mathrm{CRH}(50 \mu \mathrm{g} / \mathrm{kg}$ body weight) and/or chloroquine ( $60 \mathrm{mg} / \mathrm{kg}$ body weight) was intraperitoneally administrated from day 1 through day 6 (using saline as a vehicle). Initial body weight, DAl score, and colon length were subsequently determined by two researchers blinded to the treatment groups. In addition, inflammation in the left colon was assessed by HE staining. A-C In comparison with the DSS + vehicle group, mice in the DSS + CRH group exhibited significant increases in body weight loss, DAl scores, and colon shortening. In contrast, coadministration of chloroquine largely attenuated the aggravation effects of CRH on body weight loss and DAI Score, but no significant change in colon length ( $n=8$ per group). ${ }^{* *} P<0.01$ vs. the control group; ${ }^{\# \#} P<0.01$ vs. the DSS + vehicle group; ${ }^{\$ \$} P<0.01$ vs. the DSS + CRH group. D, E H\&E staining was used to assess histological scores in the left colon. In comparison with the DSS + vehicle group, mice in the DSS + CRH group demonstrated an aggravation of inflammatory infiltration. In contrast, coadministration of chloroquine largely alleviated the impact of CRH ( $n=8$ per group). ${ }^{* *} P<0.01$ vs. the control group; ${ }^{\#} P<0.05$ vs. the DSS + vehicle group; ${ }^{\$} P<0.05$ vs. the DSS + CRH group.

cells focally located in the lamina propria; 2 , an increase in inflammatory cells extending into the submucosa; and 3, a transmural extension of the infiltrate. Tissue damage was scored as: 1, discrete lymphoepithelial lesions; 2, mucosal erosions; and 3, extensive mucosal damage and/or extension through the deeper structures of the bowel wall. The two subscores (cell infiltration and tissue damage) were added to yield a combined histological colitis severity score ranging from 0 to 6 .

\section{Cell culture and treatment}

As outlined in a previous study, murine BMDMs were obtained through the incubation of bone marrow cells [49]. In brief, bone marrow obtained from femurs and tibias was flushed, cultured, and differentiated in bone marrow growth medium composed of Dulbecco's modified Eagle's medium (DMEM; Gibco, Grand Island, NY, USA), 10\% fetal bovine serum (FBS; Gibco, Grand Island, NY, USA), 30\% L929 cellconditioned media (source of macrophage-colony stimulating factor), and penicillin/streptomycin at $37^{\circ} \mathrm{C}$ in a humidified incubator with $5 \%$ $\mathrm{CO}_{2}$. The bone marrow growth medium was renewed every $48 \mathrm{~h}$. After 7-day cultivation, fresh medium was replaced. For additional treatments, LPS (100 ng/mL, Sigma-Aldrich, St. Louis, MO, USA), CRH $\left(10^{-8} \mathrm{M}\right.$,
Tocris, Ellisville, MO, USA), and/or chloroquine (10 $\mu \mathrm{M}$, Sigma-Aldrich, St. Louis, MO) were applied for $12 \mathrm{~h}$.

\section{Enzyme-linked immunosorbent assay (ELISA)}

TNF- $\alpha, I L-18$, and IL-1 $\beta$ levels in serum and colon tissues were quantified using commercial ELISA kits in accordance with the manufacturer's instructions (R\&D system, Minneapolis, MN, USA).

\section{Immunofluorescence staining}

Colonic tissues were fixed in $4 \%(\mathrm{w} / \mathrm{v})$ paraformaldehyde overnight and embedded in paraffin. Next, $5 \mu \mathrm{m}$ sections were cut and then processed by dewaxing and rehydration. After blocking with $5 \%$ bovine serum albumin in PBS for $2 \mathrm{~h}$, the sections were incubated with F4/80 antibody (1:100, Santa Cruz Biotechnology Inc., Dallas, Texas, USA) or LC3 antibody $\left(1: 200\right.$, Novus Biologicals, Littleton, CO, USA) overnight at $4{ }^{\circ} \mathrm{C}$. The sections were then washed three times with PBS and subsequently stained using Alexa-488- or Alexa-Cy3-labeled secondary antibody (1:500, Jackson ImmunoResearch Inc., West Grove, PA, USA) for $30 \mathrm{~min}$ at $37^{\circ} \mathrm{C}$. After washing, the sections were mounted on slides using Vectashield mounting medium containing 4',6-diamidino-2- 

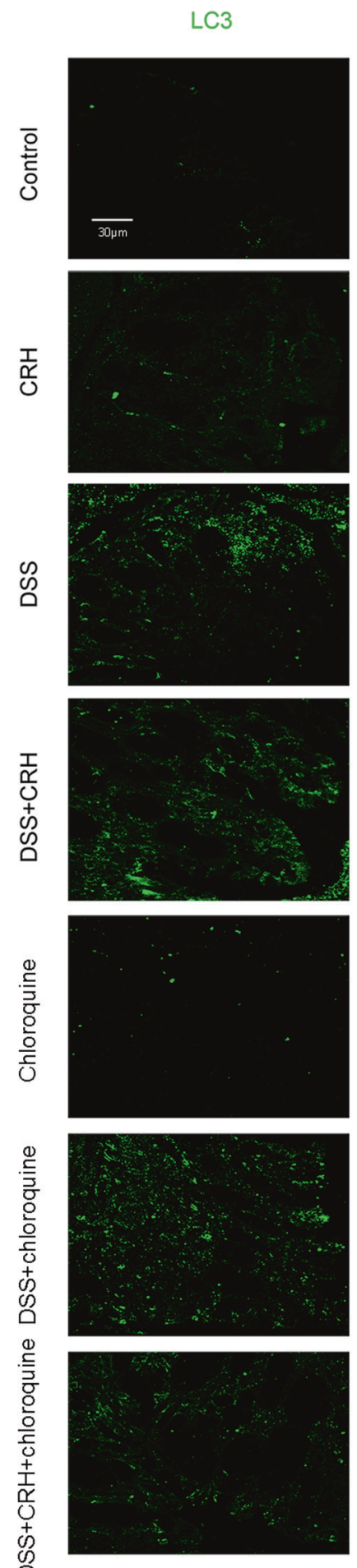

F4/80
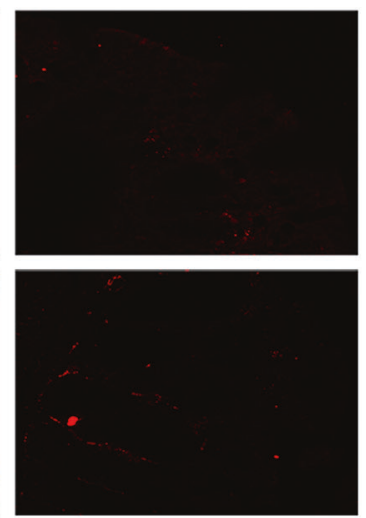

\section{DAPI}
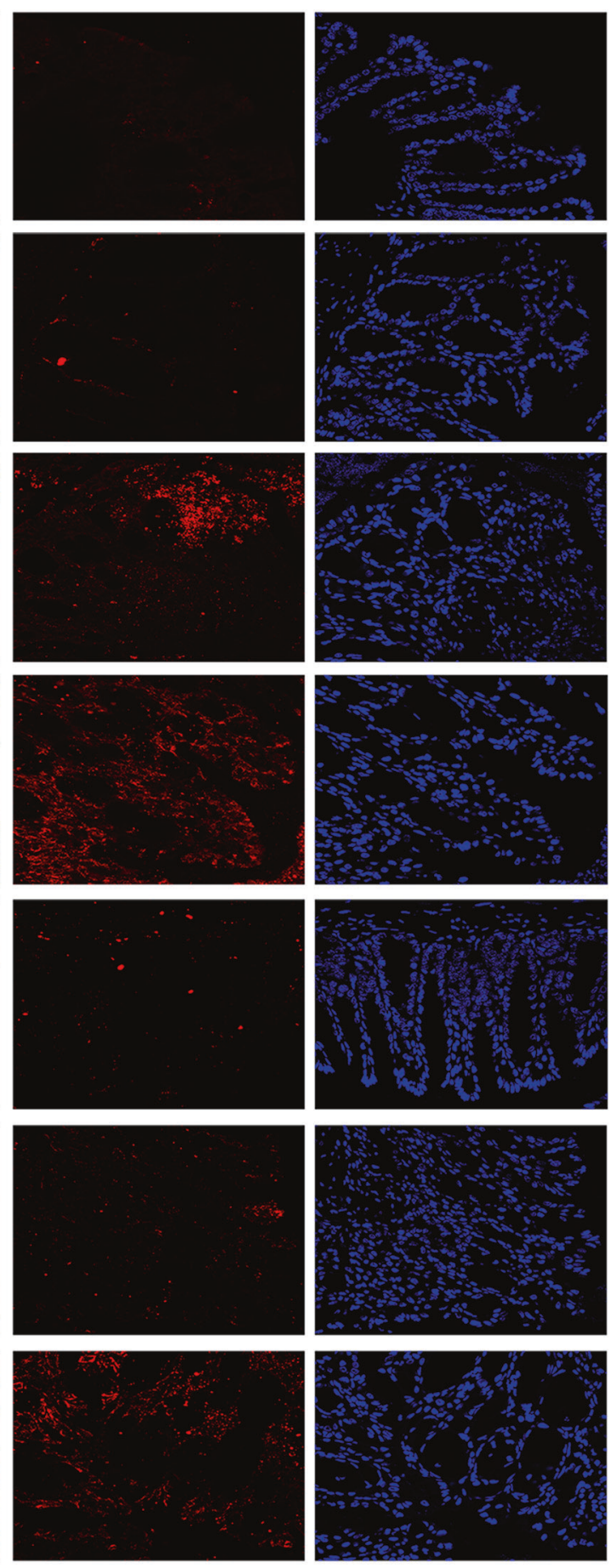

Merge
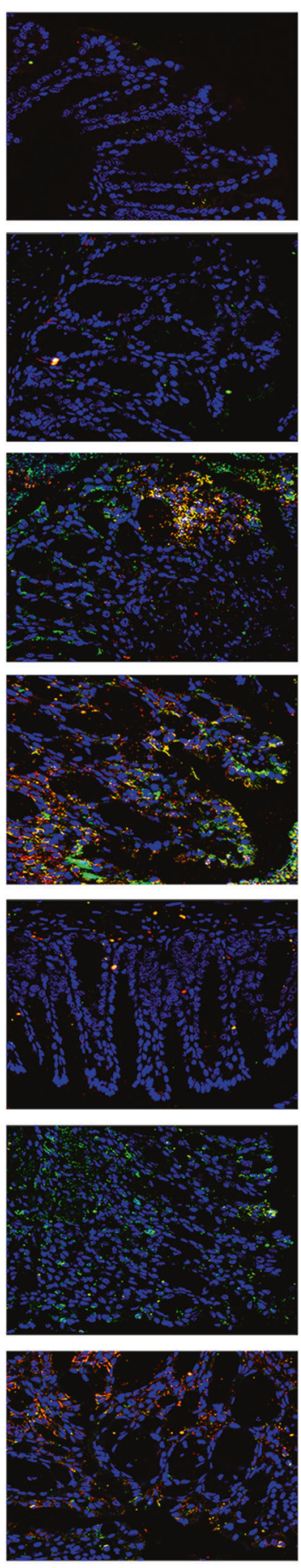

Fig. 6 Chloroquine attenuated the increase in autophagy and number of macrophages in IBD mice. C57BL/6 mice were administered DSS (3\%) for 6 days (and a control group was provided with water only for comparison). For the $\mathrm{CRH}$ and $\mathrm{CRH}$-rapamycin groups, $\mathrm{CRH}$ (50 $\mu \mathrm{g} / \mathrm{kg}$ body weight) and/or rapamycin (1.25 mg/kg body weight) was intraperitoneally injected from day 1 through day 6 using saline as a vehicle. The left edge of the colon was isolated and fixed, and immunofluorescence staining was used to assess the number of macrophages (LC3 dots) and the level of autophagy (F4/80 dots). In comparison with the DSS + vehicle group, mice in the DSS + CRH group demonstrated a significant increase in the numbers of LC3 dots and F4/80 dots. This increase was attenuated following chloroquine administration. Notably, LC3 dots colocalized obviously with the F4/80 dots.

phenylindole (Vector Laboratories, Burlingame, CA, USA). Antibody colocalization was then assessed using a confocal laser scanning microscope (Fluoview FV1000, Olympus, Tokyo, Japan). All experiments were performed in a double-blind manner.

\section{Western blotting}

Total protein was isolated from murine BMDMs and colonic tissues using a standard extraction reagent supplemented with protease inhibitor (Kangchen; Shanghai, China). The concentration of extracted 
A
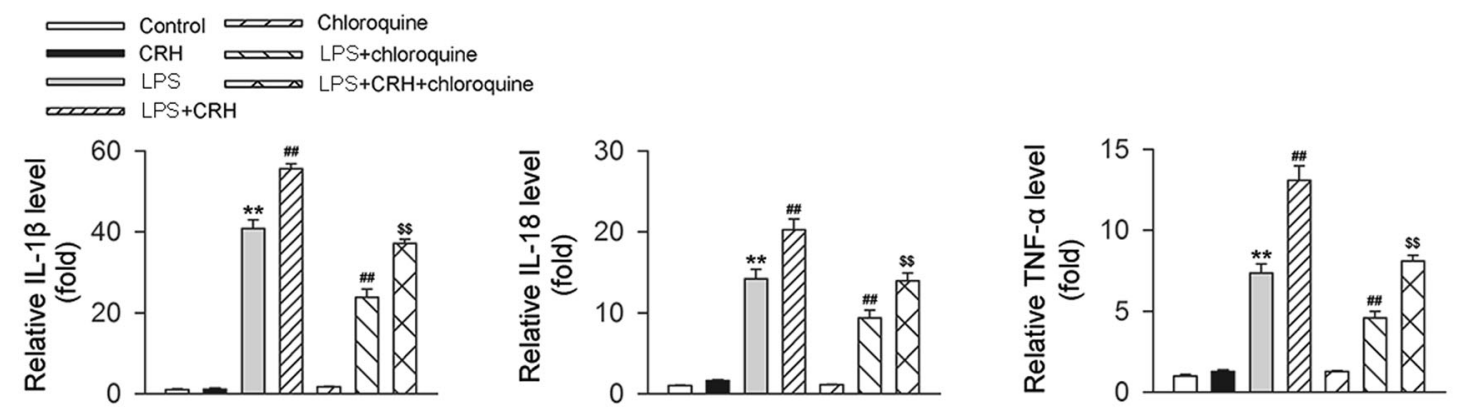

B
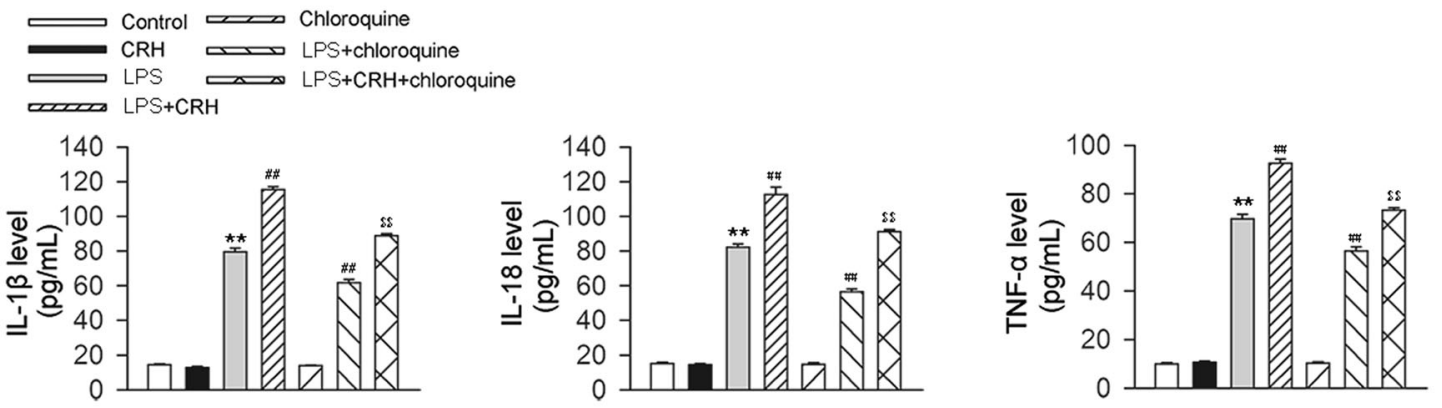

Fig. 7 Chloroquine alleviated the CRH-induced enhancement of inflammation in BMDMs under the challenge of LPS. Murine BMDMs were isolated and subsequently stimulated with LPS $(100 \mathrm{ng} / \mathrm{mL}), \mathrm{CRH}\left(10^{-8} \mathrm{M}\right)$, and/or chloroquine $(10 \mu \mathrm{M})$. Real-time qPCR and ELISA were then used to assess the levels of proinflammatory factors. In comparison with the LPS + vehicle group, CRH administration further increased the levels of IL-1 $\beta$, TNF- $\alpha$, and IL-18 mRNA (A) and protein (B). ( $n=6$ per group.) ${ }^{* *} P<0.01$ vs. the control group; ${ }^{\# \#} P<0.01$ vs. the chloroquine + vehicle group; ${ }^{\$ \$} P<0.01$ vs. the chloroquine $+\mathrm{CRH}$ group.

protein was determined using a bicinchoninic acid protein assay kit (Beyotime Institute of Biotechnology, Haimen, China). Proteins were then separated using SDS-PAGE and electro-transferred to nitrocellulose membranes as previous described [50]. Immunoblotting was performed using a Beclin-1 antibody (1:500; Cell Signaling Technology, Danvers, MA, USA), an LC3 antibody (1:500; Novus Biologicals, Littleton, CO, USA), and a p62 antibody (1:500; Cell Signaling Technology, Danvers, MA, USA). The membranes were then incubated with an IRDye800CW-conjugated secondary antibody (Rockland, Gilbertsville, $\mathrm{PA}$, USA) for $1 \mathrm{~h}$ at $25^{\circ} \mathrm{C}$. Finally, images of the blots were obtained using an Odyssey infrared imaging system (Li-Cor Biosciences, Lincoln, $N E$, USA).

\section{Real-time polymerase chain reaction (PCR)}

Total RNA was isolated from murine BMDMs and colon tissues using TRlzol (Invitrogen, Carlsbad, CA, USA). PrimeScript RT Master Mix (Takara, Otsu, Shiga, Japan) was then used for first-strand CDNA synthesis. Real-time qPCR was performed on a 7500 real-time PCR System (Applied Biosystems ${ }^{T M}$ ) using Fast Start Universal SYBR Green Master (Roche, Basel, Switzerland). The $2^{-\triangle \Delta C T}$ method was used to analyze the $\mathrm{qPCR}$ results with GAPDH as an internal reference. The sequences of the primers were as follows: TNF- $a$ (sense) 5'-AAGCCTGTAGCCCACGTCGTA-3' and (antisense) 5'-GGCA CCACTAGTTGGTTGTCTTTG-3'; IL-1 $\beta$ (sense) 5'-CTCGTGCTGTCGGA CCCCAT- $3^{\prime}$ and (antisense) 5'-AGTGTTCGTCTCGTGTTCGGAC-3'; IL-18 (sense) 5'-CAGGCCTGACATCTTCTGCAA-3' and (antisense) 5'-CTCCAGCATC AGGACAAAGAAAGCCG-3'; GAPDH (sense) 5'-GTATGACTCCACTCACGGC AAA-3' and (antisense) 5'-GGTCTCGCTCCTGGAAGATG-3'.

\section{Transmission electron microscopy}

Murine BMDMs were harvested and fixed overnight at $4{ }^{\circ} \mathrm{C}$ in $2.5 \%$ glutaraldehyde (in $0.1 \mathrm{M} \mathrm{PBS}$ ), and then postfixed in $1 \%$ buffered osmium tetroxide for $2 \mathrm{~h}$. BMDMs were then processed following routine procedures and examined under a transmission electron microscope $(\mathrm{H}-$ 700; Hitachi, Tokyo, Japan).

\section{Autophagy flux assessment}

Murine BMDMs were isolated and cultured on slides at $37^{\circ} \mathrm{C}$. When cell confluency reached $50-70 \%$, a tandem fluorescent mRFP-GFP-LC3 plasmid was transfected. In brief, after cultivation with DMEM supplemented with $10 \%$ FBS for $24 \mathrm{~h}$, murine BMDMs were incubated with plasmids for $6 \mathrm{~h}$, and then cultured in fresh DMEM supplemented with $10 \%$ FBS for $36 \mathrm{~h}$. Cellular autophagosomes $\left(\mathrm{G}^{+} \mathrm{R}^{+}\right)$and autolysosomes $\left(\mathrm{G}^{-} \mathrm{R}^{+}\right)$were subsequently imaged by confocal microscopy (Leica TCS SP8, Leica, Biberach, Germany). The total number of puncta $(>1 \mu \mathrm{m})$ per cell were counted and recorded.

\section{Colon organoid culture and treatment}

Fresh mouse and human intestinal tissues were incubated with $2 \mathrm{mmol} / \mathrm{L}$ EDTA (Gibco, USA) for $30 \mathrm{~min}$ at $4{ }^{\circ} \mathrm{C}$ and $5 \mathrm{mmol} / \mathrm{L}$ EDTA (Gibco, USA) for $30 \mathrm{~min}(\times 2)$ at $4^{\circ} \mathrm{C}$ on a roller. The tissues were then filtered and $10 \mathrm{~mL}$ of $0.1 \%$ BSA was added (Solarbio, China). Next, the isolated crypt units were plated to a density of 200 crypts per well in a $40-\mu \mathrm{L}$ droplet of Matrigel mixed with IntestiCult media. Crypt-containing Matrigel droplets were overlaid with IntestiCult complete organoid media (Stemcell Technologies, Vancouver, BC) supplemented with penicillin/streptomycin. BMDMs were then plated on the top membrane of transwell inserts for a minimum incubation of $12 \mathrm{~h}$ with LPS $(100 \mathrm{ng} / \mathrm{mL})$ stimulation. When a cell attachment of $60 \%$ was achieved, the BMDMs were cocultured with colonic organoids in the presence of LPS (100 ng/mL, refresh every $24 \mathrm{~h})$ to investigate the effect of BMDMs on the progression of IBD in vitro.

\section{Statistical analysis}

Data are presented as mean \pm SEM. The DAl scores among groups were analyzed by two-way analysis of variance (ANOVA) followed by Bonferroni's post hoc test for repeated measures. For the analysis of nonparametric data and continuous variables, a Kruskal-Wallis test followed by Dunn's post hoc test and one-way ANOVA followed by Bonferroni's post hoc test was used, respectively. A P value $<0.05$ was used to assess statistical significance. All data analyses were performed using SPSS $21.0 \mathrm{~K}$ for Windows (SPSS, Chicago, IL, USA). 
A

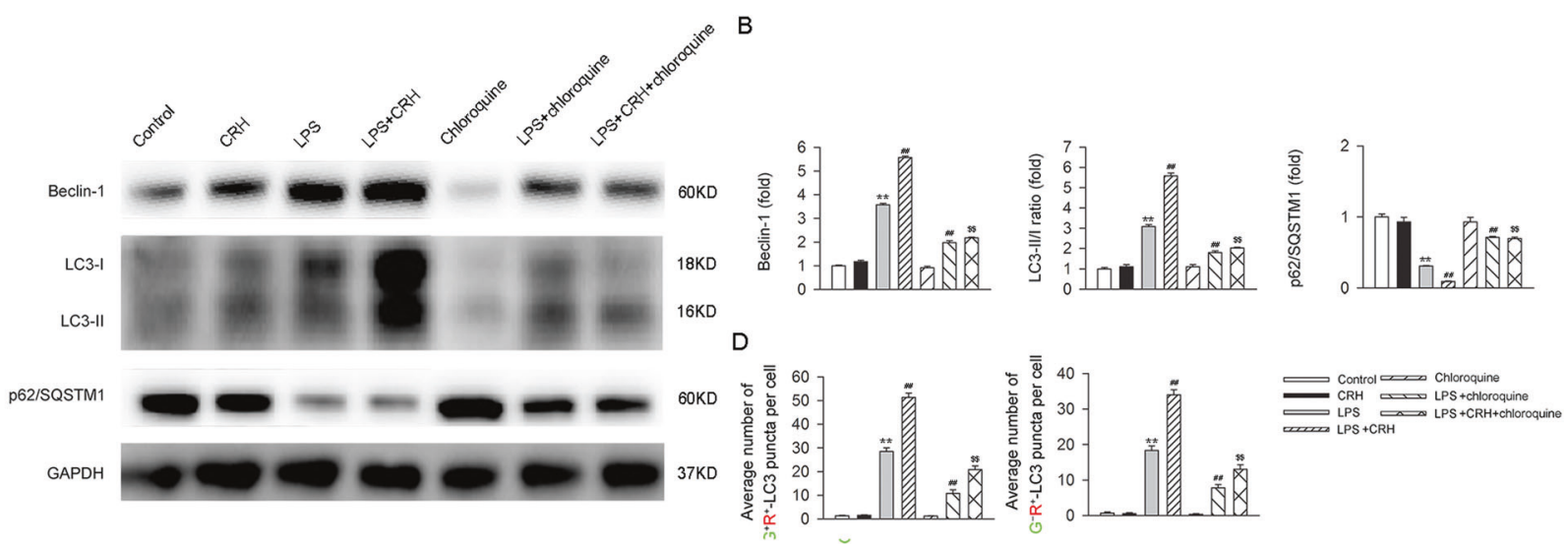

C
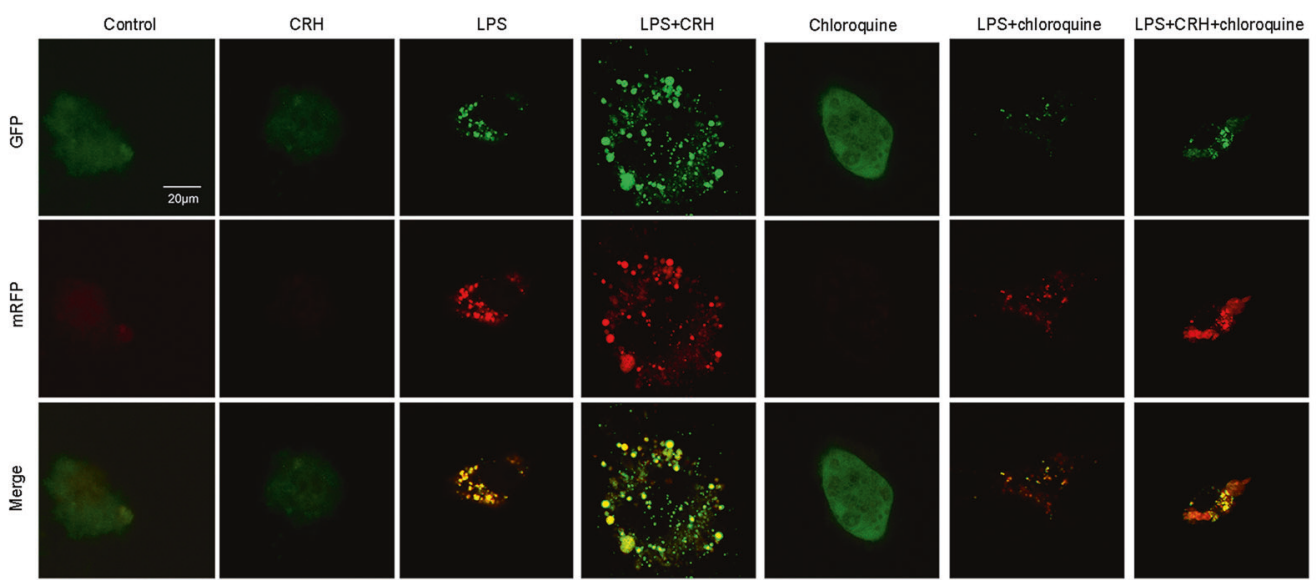

Fig. 8 Chloroquine attenuated the CRH-induced increase in autophagy in BMDMs under the challenge of LPS. BMDMs were treated with LPS $(100 \mathrm{ng} / \mathrm{mL}), \mathrm{CRH}\left(10^{-8} \mathrm{M}\right)$, and/or chloroquine $(10 \mu \mathrm{M})$. Representative images of mRFP-GFP-LC3-transfected BMDMs were then assessed by immunofluorescence. A, B In comparison with the LPS + vehicle group, mice in the LPS + CRH group exhibited a significant increase in Beclin-1 and the LC3-II/I ratio, but a decrease in p62. These effects of CRH were blocked by chloroquine administration. ( $n=5$ per group). ${ }^{* *} P<0.01$ vs. the control group; ${ }^{\# \#} P<0.01$ vs. the LPS + vehicle group; ${ }^{\$} P<0.01$ vs. the LPS + CRH group. C While CRH significantly elevated autophagy levels under the challenge of LPS, chloroquine administration attenuated the effects of CRH. D Quantitative analysis of the number of yellow autophagosomes and red autolysosomes. ( $n=6$ per group). ${ }^{* *} P<0.01$ vs. the control group; ${ }^{\# \#} P<0.01$ vs. the LPS + vehicle group; $\$ \$ P<0.01$ vs. the LPS + CRH group.

\section{DATA AVAILABILITY}

Data underlying this article will be shared on reasonable request to the corresponding author.

\section{REFERENCES}

1. Palmela C, Chevarin C, Xu Z, Torres J, Sevrin G, Hirten R, et al. Adherent-invasive Escherichia coli in inflammatory bowel disease. Gut. 2018;67:574-87.

2. Singh S, Al-Darmaki A, Frolkis AD, Seow CH, Leung $Y$, Novak $K L$, et al. Postoperative mortality among patients with inflammatory bowel diseases: a systematic review and meta-analysis of population-based studies. Gastroenterology. 2015;149:928-37.

3. Regueiro M, Greer JB, Szigethy E. Etiology and treatment of pain and psychosocial issues in patients with inflammatory bowel diseases. Gastroenterology. 2017;152:430-9.e4.

4. Ventham NT, Kennedy NA, Nimmo ER, Satsangi J. Beyond gene discovery in inflammatory bowel disease: the emerging role of epigenetics. Gastroenterology. 2013;145:293-308.

5. Leber A, Hontecillas R, Tubau-Juni N, Zoccoli-Rodriguez V, Abedi V, BassaganyaRiera J. NLRX1 modulates immunometabolic mechanisms controlling the hostgut microbiota interactions during inflammatory bowel disease. Front Immunol. 2018;9:363.

6. Sina C, Kemper C, Derer S. The intestinal complement system in inflammatory bowel disease: shaping intestinal barrier function. Semin Immunol. 2018;37:66-73.

7. Uo M, Hisamatsu T, Miyoshi J, Kaito D, Yoneno K, Kitazume MT, et al. Mucosal CXCR4+ IgG plasma cells contribute to the pathogenesis of human ulcerative colitis through FcgammaR-mediated CD14 macrophage activation. Gut. 2013;62:1734-44.

8. Shao BZ, Wang SL, Pan P, Yao J, Wu K, Li ZS, et al. Targeting NLRP3 inflammasome in inflammatory bowel disease: putting out the fire of inflammation. Inflammation. 2019;42:1147-59.

9. Brzozowski B, Mazur-Bialy A, Pajdo R, Kwiecien S, Bilski J, Zwolinska-Wcislo M, et al. Mechanisms by which stress affects the experimental and clinical inflammatory bowel disease (IBD): role of brain-gut axis. Curr Neuropharmacol. 2016;14:892-900.

10. Gracie DJ, Ford AC. A bidirectional relationship between symptom reporting and perceived stress, but not disease activity, in inflammatory bowel disease: more questions than answers? Gastroenterology. 2017;153:1444-5.

11. Guerrero-Alba R, Valdez-Morales EE, Jimenez-Vargas NN, Lopez-Lopez C, JaramilloPolanco J, Okamoto T, et al. Stress activates pronociceptive endogenous opioid signalling in DRG neurons during chronic colitis. Gut. 2017;66:2121-31.

12. Gavin KM, Shea KL, Gibbons E, Wolfe P, Schwartz RS, Wierman ME, et al. Gonadotropin releasing hormone agonist in premenopausal women does not alter hypothalamic-pituitary-adrenal axis response to corticotropin-releasing hormone. Am J Physiol Endocrinol Metab. 2018;315:E316-25.

13. Armario A. Activation of the hypothalamic-pituitary-adrenal axis by addictive drugs: different pathways, common outcome. Trends Pharmacol Sci. 2010;31:318-25.

14. Ji NN, Kang J, Hua R, Zhang YM. Involvement of dopamine system in the regulation of the brain corticotropin-releasing hormone in paraventricular nucleus in a rat model of chronic visceral pain. Neurol Res. 2018;40:650-7.

15. Donelan J, Boucher W, Papadopoulou N, Lytinas M, Papaliodis D, Dobner P, et al. Corticotropin-releasing hormone induces skin vascular permeability through a neurotensin-dependent process. Proc Natl Acad Sci USA. 2006;103:7759-64. 
16. van Tol EA, Petrusz P, Lund PK, Yamauchi M, Sartor RB. Local production of corticotropin releasing hormone is increased in experimental intestinal inflammation in rats. Gut. 1996;39:385-92.

17. Tache Y, Bonaz B. Corticotropin-releasing factor receptors and stress-related alterations of gut motor function. J Clin Investig. 2007;117:33-40.

18. Chang J, Hoy JJ, Idumalla PS, Clifton MS, Pecoraro NC, Bhargava A. Urocortin 2 expression in the rat gastrointestinal tract under basal conditions and in chemical colitis. Peptides. 2007;28:1453-60.

19. Kimura $T$, Amano $T$, Uehara $H$, Ariga $H$, Ishida $T$, Torii $A$, et al. Urocortin I is present in the enteric nervous system and exerts an excitatory effect via cholinergic and serotonergic pathways in the rat colon. Am J Physiol Gastrointest Liver Physiol. 2007;293:G903-10.

20. Wallon C, Yang PC, Keita AV, Ericson AC, McKay DM, Sherman PM, et al. Corticotropin-releasing hormone $(\mathrm{CRH})$ regulates macromolecular permeability via mast cells in normal human colonic biopsies in vitro. Gut. 2008;57:50-8.

21. Kokkotou E, Torres D, Moss AC, O'Brien M, Grigoriadis DE, Karalis $K$, et al. Corticotropin-releasing hormone receptor 2-deficient mice have reduced intestinal inflammatory responses. J Immunol. 2006;177:3355-61.

22. Gravanis A, Margioris AN. The corticotropin-releasing factor (CRF) family of neuropeptides in inflammation: potential therapeutic applications. Curr Med Chem. 2005;12:1503-12.

23. Im E, Rhee SH, Park YS, Fiocchi C, Tache Y, Pothoulakis C. Corticotropin-releasing hormone family of peptides regulates intestinal angiogenesis. Gastroenterology. 2010;138:2457-67.e1-5.

24. Levine B, Kroemer G. Biological functions of autophagy genes: a disease perspective. Cell. 2019;176:11-42.

25. Clarke AJ, Simon AK. Autophagy in the renewal, differentiation and homeostasis of immune cells. Nat Rev Immunol. 2019;19:170-83.

26. Corona AK, Jackson WT. Finding the middle ground for autophagic fusion requirements. Trends Cell Biol. 2018;28:869-81.

27. Chu $H$, Khosravi A, Kusumawardhani IP, Kwon AH, Vasconcelos AC, Cunha LD, et al. Gene-microbiota interactions contribute to the pathogenesis of inflammatory bowel disease. Science. 2016;352:1116-20.

28. Lee HY, Kim J, Quan W, Lee JC, Kim MS, Kim SH, et al. Autophagy deficiency in myeloid cells increases susceptibility to obesity-induced diabetes and experimental colitis. Autophagy. 2016;12:1390-403.

29. Enayat S, Seyma Ceyhan M, Taskoparan B, Stefek M, Banerjee S. CHNQ, a novel 2Chloro-1,4-naphthoquinone derivative of quercetin, induces oxidative stress and autophagy both in vitro and in vivo. Arch Biochem Biophys. 2016;596:84-98.

30. Wang Z, Shi L, Hua S, Qi C, Fang M. IL-33 ameliorates experimental colitis involving regulation of autophagy of macrophages in mice. Cell Biosci. 2019;9:10.

31. Vanuytsel $T$, van Wanrooy $S$, Vanheel $H$, Vanormelingen $C$, Verschueren $S$, Hou-

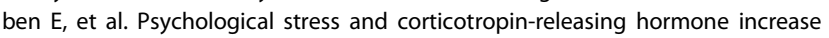
intestinal permeability in humans by a mast cell-dependent mechanism. Gut. 2014;63:1293-9.

32. Wang SL, Shao BZ, Zhao SB, Chang $X$, Wang $P$, Miao $C Y$, et al. Intestinal autophagy links psychosocial stress with gut microbiota to promote inflammatory bowel disease. Cell Death Dis. 2019;10:391.

33. Tschurtschenthaler M, Adolph TE, Ashcroft JW, Niederreiter L, Bharti R, Saveljeva $S$, et al. Defective ATG16L1-mediated removal of IRE1alpha drives Crohn's disease-like ileitis. J Exp Med. 2017;214:401-22.

34. Kawahito Y, Sano H, Mukai S, Asai K, Kimura S, Yamamura Y, et al. Corticotropin releasing hormone in colonic mucosa in patients with ulcerative colitis. Gut. 1995;37:544-51.

35. La JH, Sung TS, Kim HJ, Kim TW, Kang TM, Yang IS. Peripheral corticotropin releasing hormone mediates post-inflammatory visceral hypersensitivity in rats. World J Gastroenterol. 2008;14:731-6.

36. Gay J, Kokkotou E, O'Brien M, Pothoulakis C, Karalis KP. Corticotropin-releasing hormone deficiency is associated with reduced local inflammation in a mouse model of experimental colitis. Endocrinology. 2008;149:3403-9.

37. Chen $H$, Shi H, Liu Y, Ren $X$, He S, Chang $X$, et al. Activation of corticotropinreleasing factor receptor 1 aggravates dextran sodium sulphate-induced colitis in mice by promoting M1 macrophage polarization. Mol Med Rep. 2018;17:234-42.

38. Teitelbaum AA, Gareau MG, Jury J, Yang PC, Perdue MH. Chronic peripheral administration of corticotropin-releasing factor causes colonic barrier dysfunction similar to psychological stress. Am J Physiol Gastrointest Liver Physiol. 2008;295:G452-9.

39. Saito-Nakaya K, Hasegawa R, Nagura Y, Ito H, Fukudo S. Corticotropin-releasing hormone receptor 1 antagonist blocks colonic hypersensitivity induced by a combination of inflammation and repetitive colorectal distension. Neurogastroenterol Motil. 2008;20:1147-56.

40. Shao BZ, Han BZ, Zeng YX, Su DF, Liu C. The roles of macrophage autophagy in atherosclerosis. Acta Pharmacol Sin. 2016;37:150-6.

41. Gatica D, Chiong M, Lavandero S, Klionsky DJ. Molecular mechanisms of autophagy in the cardiovascular system. Circ Res. 2015;116:456-67.
42. Shen T, Li S, Cai LD, Liu JL, Wang CY, Gan WJ, et al. Erbin exerts a protective effect against inflammatory bowel disease by suppressing autophagic cell death. Oncotarget. 2018;9:12035-49.

43. Nagar J, Ranade S, Kamath V, Singh S, Karunanithi P, Subramani S, et al. Therapeutic potential of chloroquine in a murine model of inflammatory bowel disease. Int Immunopharmacol. 2014;21:328-35.

44. Glinsky GV. SNP-guided microRNA maps (MirMaps) of 16 common human disorders identify a clinically accessible therapy reversing transcriptional aberrations of nuclear import and inflammasome pathways. Cell Cycle. 2008;7:3564-76.

45. Goenka MK, Kochhar R, Tandia B, Mehta SK. Chloroquine for mild to moderately active ulcerative colitis: comparison with sulfasalazine. Am J Gastroenterol. 1996;91:917-21.

46. Kim TW, Shin JS, Chung KS, Lee YG, Baek NI, Lee KT. Anti-inflammatory mechanisms of koreanaside $A$, a lignan isolated from the Flower of Forsythia koreana, against LPS-induced macrophage activation and DSS-induced colitis mice: the crucial role of AP-1, NF-kappaB, and JAK/STAT signaling. Cells. 2019;8:1163.

47. Torretta S, Scagliola A, Ricci L, Mainini F, Di Marco S, Cuccovillo I, et al. D-mannose suppresses macrophage IL-1beta production. Nat Commun. 2020;11:6343.

48. Wirtz S, Neufert C, Weigmann B, Neurath MF. Chemically induced mouse models of intestinal inflammation. Nat Protoc. 2007;2:541-6.

49. Choi SH, Gonen A, Diehl CJ, Kim J, Almazan F, Witztum JL, et al. SYK regulates macrophage MHC-II expression via activation of autophagy in response to oxidized LDL. Autophagy. 2015;11:785-95.

50. Shao BZ, Wei W, Ke P, Xu ZQ, Zhou JX, Liu C. Activating cannabinoid receptor 2 alleviates pathogenesis of experimental autoimmune encephalomyelitis via activation of autophagy and inhibiting NLRP3 inflammasome. CNS Neurosci Ther. 2014:20:1021-8.

\section{ACKNOWLEDGEMENTS}

YB was supported by the National Natural Science Foundation of China (81873546 and 82170567), "Shu Guang" project of Shanghai Municipal Education Commission and Shanghai Education Development Foundation (No. 19SG30), the National Key R\&D Program of China (No. 2018YFC1313103), and 234 Discipline Climbing Plan of Changhai Hospital, Naval Medical University (No. 2019YXK004). S-LW was supported by the National Natural Science Foundation of China (No. 82100587), the Shanghai Sailing Program (No. 21YF1458700), and the Youth Start-up Fund of Changhai Hospital, Naval Medical University (No. 2019QNB02). TX was supported by the National Natural Science Foundation of China (No. 81702373). XF was supported by the National Natural Science Foundation of China (No. 81800479).

\section{AUTHOR CONTRIBUTIONS}

S-BZ, J-YW, Z-XH, Y-HS, and XC performed the majority of experiments, analyzed data, and wrote the manuscript. TX and XF performed some cell experiments. ZSL and CX designed and supervised the study. S-LW and YB formulated the hypothesis, supervised the study, and performed manuscript review and editing.

\section{COMPETING INTERESTS}

The authors declare no competing interests.

\section{ETHICS APPROVAL AND CONSENT TO PARTICIPATE}

This study was performed in accordance with the Declaration of Helsinki and approved by the Medical Ethics Committee in Shanghai Changhai Hospital, Shanghai, China. Written informed consent was obtained from all the participants before enrollment. Animal procedures were in accordance with the guidelines of the Animal Care Committee of Navy Medical University, Shanghai, China.

\section{ADDITIONAL INFORMATION}

Supplementary information The online version contains supplementary material available at https://doi.org/10.1038/s41420-021-00767-8.

Correspondence and requests for materials should be addressed to Zhao-Shen Li, Can Xu, Shu-Ling Wang or Yu Bai.

Reprints and permission information is available at http://www.nature.com/ reprints 
Publisher's note Springer Nature remains neutral with regard to jurisdictional claims in published maps and institutional affiliations.

Open Access This article is licensed under a Creative Commons Attribution 4.0 International License, which permits use, sharing, BY Attribution 4.0 International License, which permits use, sharing,
adaptation, distribution and reproduction in any medium or format, as long as you give appropriate credit to the original author(s) and the source, provide a link to the Creative
Commons license, and indicate if changes were made. The images or other third party material in this article are included in the article's Creative Commons license, unless indicated otherwise in a credit line to the material. If material is not included in the article's Creative Commons license and your intended use is not permitted by statutory regulation or exceeds the permitted use, you will need to obtain permission directly from the copyright holder. To view a copy of this license, visit http://creativecommons. org/licenses/by/4.0/.

C The Author(s) 2021 